\title{
Clear cell neuroendocrine tumor G1 of the gallbladder without von Hippel-Lindau disease
}

\author{
MITSUAKI ISHIDA $^{1}$, HISANORI SHIOMI ${ }^{2}$, SHIGEYUKI NAKA ${ }^{2}$, TOHRU TANI $^{2}$ and HIDETOSHI OKABE ${ }^{1}$ \\ ${ }^{1}$ Department of Clinical Laboratory Medicine and Division of Diagnostic Pathology; \\ ${ }^{2}$ Department of Surgery, Shiga University of Medical Science, Otsu, Shiga 520-2192, Japan
}

Received June 22, 2012; Accepted August 29, 2012

DOI: 10.3892/ol.2012.899

\begin{abstract}
Clear cell neuroendocrine tumor (NET) is a rare but distinct histopathological variant of NET most often observed in patients with von Hippel-Lindau disease (VHL). Clear cell NET in non-VHL patients is extremely rare and this report is only the second to describe a case of clear cell NET G1 (carcinoid tumor) of the gallbladder without VHL. A 71-year-old male without past or family history of VHL presented with a polypoid lesion in the fundus of the gallbladder and laparoscopic cholecystectomy was performed. Histopathological study revealed that the polypoid lesion was comprised of nests or trabecular growths of clear cells without atypia. Immunohistochemically, the neoplastic cells were diffusely positive for endocrine markers, but negative for $\alpha$-inhibin. Clinicopathological review of cases with clear cell NET G1 of the gallbladder revealed that this disease occurs in patients with or without VHL, but that gallbladder stones and cholecystitis were present in non-VHL cases. We hypothesized that the occurrence of clear cell NET of the gallbladder (particularly non-VHL cases) may be associated with chronic cholecystitis induced by gallbladder stones. Moreover, $\alpha$-inhibin was detected in clear cell NET tumor cells in one VHL case, but not in two non-VHL cases. These findings suggest that $\alpha$-inhibin expression is a useful determinant of an association between clear cell NET and VHL.
\end{abstract}

\section{Introduction}

Neuroendocrine tumors (NETs) are relatively rare (1). Previous World Health Organization Classification groups NETs into G1 (referred to synonymously as 'carcinoid tumor'), G2 and G3 (neuroendocrine carcinoma) (2). Primary NETs of the gallbladder comprise only $0.5 \%$ of all NETs arising from any tissue or organ and less than $2 \%$ of all cases of gallbladder cancer (3).

Correspondence to: Dr Mitsuaki Ishida, Department of Clinical Laboratory Medicine and Division of Diagnostic Pathology, Shiga University of Medical Science, Tsukinowa-cho, Seta, Otsu, Shiga 520-2192, Japan

E-mail: mitsuaki@belle.shiga-med.ac.jp

Key words: neuroendocrine tumor, carcinoid tumor, clear cell, gallbladder, von Hippel-Lindau disease
Moreover, the majority of primary gallbladder NETs are G3, making a primary NET G1 in this organ extremely rare $(3,4)$.

The cytoplasm of NET cells is usually eosinophilic to amphophilic; however, in rare cases, the cytoplasm appears clear and is referred to as 'clear cell variant'. This variant has been described as a distinctive manifestation of von Hippel-Lindau disease (VHL) (5) and there are few reports of clear cell NET in non-VHL patients (6). Only two cases of primary gallbladder clear cell NET G1 have been reported in the English-language literature: one was associated with VHL (7) and the other was not (8). This report describes a second case of clear cell NET G1 of the gallbladder in a patient without VHL and discusses the clinicopathological features of this extremely rare lesion.

\section{Patient and methods}

Patient. A 71-year-old Japanese male without past or family history of VHL, presented with sudden abdominal pain. Computed tomography demonstrated stones in the common bile duct and gallbladder, as well as a 9-mm polypoid lesion in the fundus of the gallbladder (Fig. 1). No tumorous lesions were detected in the pancreas or kidneys and no symptoms of carcinoid syndrome were noted. Laparoscopic cholecystectomy was performed. The study was approved by the ethics committee of Shiga University of Medical Science, Shiga, Japan. Written informed patient consent was obtained from the patient.

Materials and methods. Formalin-fixed, paraffin-embedded tissue blocks of the resected specimen of the gallbladder were cut into $3-\mu \mathrm{m}$ thick sections, deparaffinized and rehydrated. Each section was stained with hematoxylin and eosin and then used for immunostaining. Immunohistochemical analyses were performed using an autostainer (XT system Benchmark, Ventana Medical System, Tucson, AZ, USA) according to the manufacturer's instructions. The following primary antibodies were used: mouse monoclonal anti- $\alpha$-inhibin (R1, Thermo Fisher Scientific, Waltham, MA, USA), mouse monoclonal anti$\alpha$-internexin (2E3, Lab Vision, Waltham, MA, USA), mouse monoclonal anti-chromogranin A (DAK-A3), rabbit polyclonal anti-gastrin, rabbit polyclonal anti-glucagon (all purchased from Dako Cytomation, Glostrup, Denmark), mouse monoclonal anti-insulin (Z006, Nichirei Bioscience, Tokyo, Japan), mouse monoclonal anti-Ki-67 (MM1, Novocastra Laboratories, Ltd., Newcastle-upon-Tyne, UK), mouse monoclonal anti-peripherin 
Table I. Clinicopathological features of clear cell neuroendocrine tumor G1 of the gallbladder.

\begin{tabular}{lllcccc}
\hline Case & Age/Gender & Location & VHL & Cholecystitis & Gallbladder stone & Reference \\
\hline 1 & 38/Male & Neck & + & Not available & - & 7 \\
2 & 64/Male & Neck & - & + & + & + \\
Present & 71/Male & Fundus & - & + & + \\
\hline
\end{tabular}

VHL, von Hippel-Lindau disease.

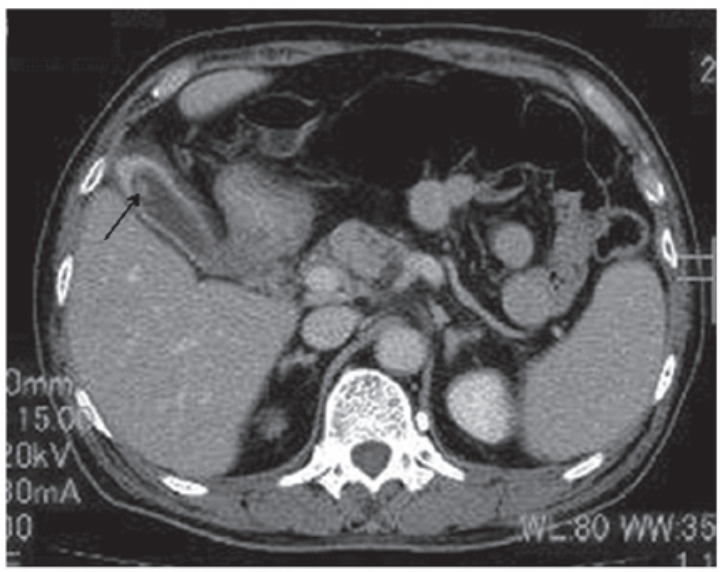

Figure 1. Abdominal CT. Polypoid lesion in the fundus of the gallbladder (arrow). CT, computed tomography.

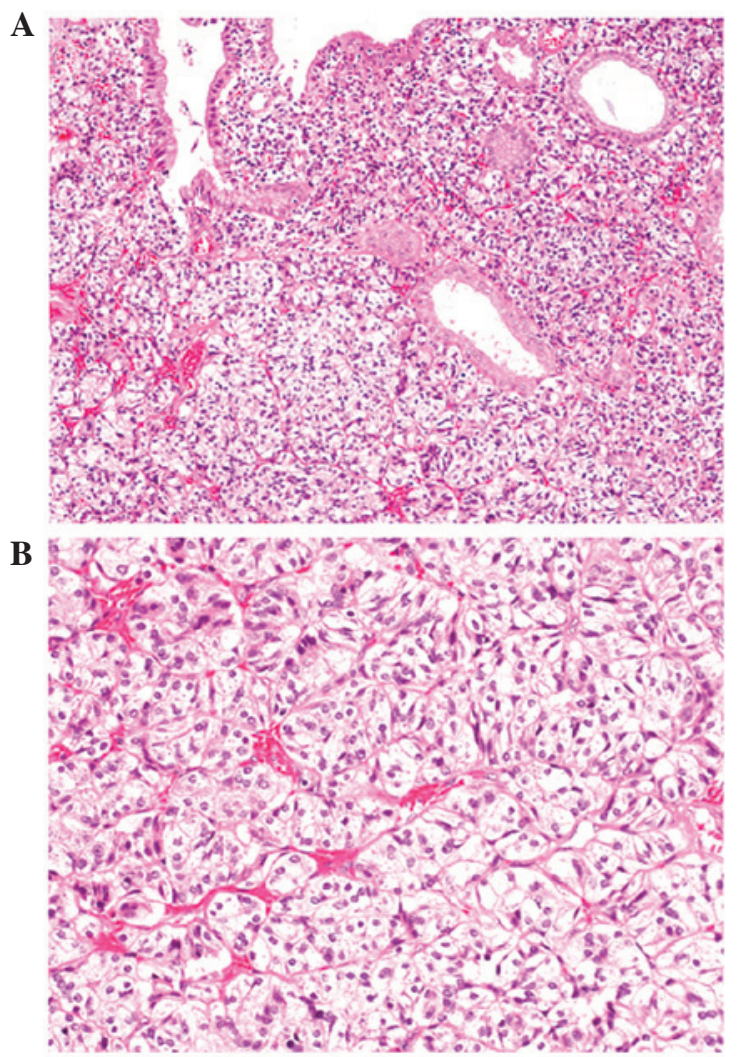

Figure 2. Histopathological analysis of polyp of the gallbladder. (A) Nests or trabecular growths of clear cells separated by delicate capillary networks. Entrapped non-neoplastic biliary glands are present among clear cell nests (hematoxylin and eosin stain, x100). (B) Clear cells have small round nuclei with inconspicuous nucleoli (hematoxylin and eosin stain, $\mathrm{x} 400$ ).

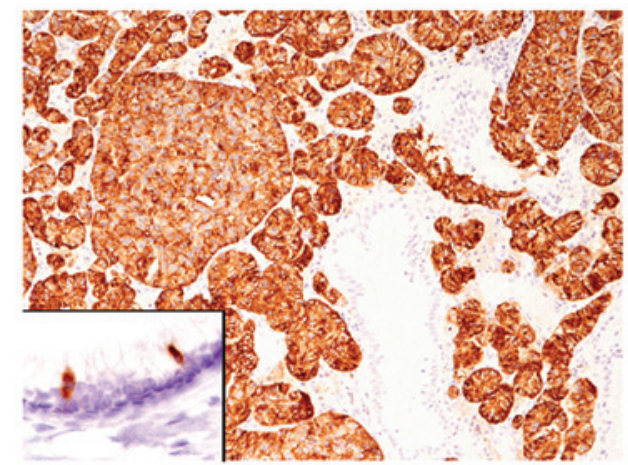

Figure 3. Immunohistochemical analysis of chromogranin A in clear cells. Chromogranin A is diffusely expressed in clear cells (x100). A few chromogranin A-positive cells are present in the non-neoplastic glands (inset; $\mathrm{x} 400$ ).

(PJM50, Novocastra), rabbit polyclonal anti-pancreatic polypeptide (Lab Vision), rabbit polyclonal anti-S-100 protein (Nichirei), mouse monoclonal anti-serotonin (5HT-H209, Dako), rabbit polyclonal anti-somatostatin (Dako) and mouse monoclonal anti-synaptophysin (27G12, Novocastra).

\section{Results}

Macroscopically, we observed three small gallbladder stones in the lumen, a mild thickening in the wall of the gallbladder and a 9-mm yellowish polypoid lesion in the fundus.

Histopathologically, the polypoid lesion was comprised of nests or trabecular growths of cuboidal cells. These cells had rich clear cytoplasm and small round nuclei with inconspicuous nucleoli separated by delicate capillary networks (Fig. 2A and B). Mitotic figures were rarely observed $(<1 / 20$ high power fields). The neoplastic growth of clear cells was restricted to the lamina propria of the polypoid lesion. The surface of the polyp was composed of biliary epithelium without atypia and non-neoplastic biliary glands were entrapped among the nests of clear cells (Fig. 2B). Neither vascular nor lymphatic invasion were present. Chronic cholecystitis with Rokitansky-Aschoff sinuses, as well as gastric metaplasia and adenomyosis, was observed in the surrounding gallbladder tissue, however, intestinal metaplasia was not noted.

Immunohistochemical analyses revealed diffuse expression of synaptophysin and chromogranin A in the neoplastic clear cells (Fig. 3), but no S-100 protein or $\alpha$-inhibin. While somatostatin was focally expressed in clear cells, we did not detect any immunoreactivity for gastrin, glucagon, insulin, serotonin or pancreatic polypeptide. The $\mathrm{Ki}-67$ labeling index was $0.8 \%$. The expression of peripherin and $\alpha$-internexin was 
not observed in the neoplastic cells. In addition, we detected focal expression of chromogranin A and synaptophysin in the non-neoplastic glands with gastric metaplasia (Fig. 3, inset).

According to the histopathological and immunohistochemical findings of the present study, diagnosis of clear cell NET G1 of the gallbladder was made.

\section{Discussion}

In the present study we describe the second reported case of clear cell NET G1 of the gallbladder in a patient without VHL. Table I summarizes the clinicopathological features of all three reported cases of clear cell NET G1 of the gallbladder $(7,8)$. All patients were male and middle-aged to elderly (average age, 57.7 years; range, 38-71). Lesions were located in the neck in the two previous cases and in the fundus in the present study. Only one previous case was associated with VHL and the others, including the present case, were not associated with VHL. Cases of clear cell NET G1 of the gallbladder all occurred in males, contrasting with general cases of clear cell NET G1 of the pancreas and gallbladder NETs, which are more prevalent in females $(60-70 \%)(3,5)$.

While normal gallbladder mucosa has no neuroendocrine cells, gallbladder mucosa with gastric and/or intestinal metaplasia contains neuroendocrine cells that express peptides, including gastrin, somatostatin, serotonin and glucagon (9-11). Moreover, the majority of previous reports of NETs of the gallbladder describe cases with gallbladder stones and cholecystitis $(3,12)$. In one study, intestinal metaplasia was found in $11.7 \%$ of gallbladders with cholelithiasis and $83.3 \%$ also contained chromogranin A-positive neuroendocrine cells (11). These data suggest that the occurrence of gallbladder NETs is associated with chronic cholecystitis induced by gallbladder stones and that this association may explain the predilection for gallbladder NETs in females.

Cholecystitis was also evident in two cases of clear cell NET G1 of the gallbladder. While gastric metaplasia with chromogranin A-positive neuroendocrine cells was observed in the present case, it is unclear whether neuroendocrine cells were present in gallbladder mucosa in the case reported by Konishi et al (8). Although reported cases are extremely limited, gallbladder stones were also found in two of three clear cell NET G1 cases reviewed here (the case without gallbladder stone was VHL-related; Table I). Therefore, chronic cholecystitis induced by gallbladder stones may be associated with clear cell NET G1 (particularly non-VHL-related cases) as well as conventional gallbladder NETs. It is plausible that gallbladder NETs, including the clear cell variant, may originate from neuroendocrine cells of intestinal and/or gastric metaplastic mucosa induced by chronic cholecystitis (3).

$\alpha$-inhibin is expressed in NET G1 of the gallbladder and pancreas associated with VHL. However, classical NET G1 of the gallbladder and non-VHL associated NET of the pancreas do not exhibit positive immunoreactivity for this marker and $\alpha$-inhibin has subsequently been reported as a marker for VHL (7). In the present case of clear cell NET G1, consistent with the previous report without VHL (8), no $\alpha$-inhibin was observed in the tumor cells. These results suggest that surveillance of $\alpha$-inhibin expression may be a useful criterion for distinguishing whether clear cell NET is associated with VHL.
We previously characterized the expression of neuronal intermediate filament proteins in NETs of various organs $(13,14)$. While peripherin (a type III intermediate filament protein expressed in normal peripheral nerves) is expressed in all NET G1 of the rectum, its expression incidence is low in NET G2 of the rectum (13). By contrast, expression of $\alpha$-internexin (a type IV intermediate filament protein normally found in the central nervous system) is observed in all NET G1 of the appendix and approximately half of rectal NET G1. All appendiceal NET G1 cases co-express peripherin and $\alpha$-internexin (14). Since neither peripherin nor $\alpha$-internexin expression was observed in this case of clear cell NET G1 of the gallbladder, it appears that intermediate filament protein expression varies with NET origin.

In conclusion, while clear cell NET is a rare histopathological variant often described as a distinct manifestation of VHL, we report a rare case of clear cell NET G1 of the gallbladder without VHL. Surveillance immunohistochemistry for $\alpha$-inhibin may prove useful as a determinant of whether a clear cell NET is VHL-associated or not.

\section{References}

1. Massironi S, Sciola V, Peracchi M, Ciafardini C, Spampatti MP and Conte D: Neuroendocrine tumors of the gastro-enteropancreatic system. World J Gastroenterol 14: 5377-5384, 2008.

2. Komminoth P, Arnold R, Capella C, et al: Neuroendocrine neoplasms of the gallbladder and extrahepatic bile ducts. In: WHO Classification of Tumours of the Digestive System. Bosman FT, Carneiro F, Hruban RH and Theise ND (eds). IARC Press, Lyon, pp274-276, 2010.

3. Eltawil KM, Gustafsson BI, Kidd M and Modlin IM: Neuroendocrine tumors of the gallbladder: an evaluation and reassessment of management strategy. J Clin Gastroenterol 44: 687-695, 2010.

4. Anjaneyulu V, Shankar-Swarnalatha G and Rao SC: Carcinoid tumor of the gall bladder. Ann Diagn Pathol 11: 113-116, 2007.

5. Hoang MP, Hruban RH and Albores-Saavedra J: Clear cell endocrine pancreatic tumor mimicking renal cell carcinoma: a distinctive neoplasm of von Hippel-Lindau disease. Am J Surg Pathol 25: 602-609, 2001.

6. Nunobe S, Fukushima N, Yachida S, Shimada K, Kosuge T and Sakamoto M: Clear cell endocrine tumor of the pancreas which is not associated with von Hippel-Lindau disease: report of a case. Surg Today 33: 470-474, 2003.

7. Sinkre PA, Murakata L, Rabin L, Hoang MP and AlboresSaavedra J: Clear cell carcinoid tumor of the gallbladder: another distinctive manifestation of von Hippel-Lindau disease. Am J Surg Pathol 25: 1334-1339, 2001

8. Konishi E, Nakashima Y, Smyrk TC and Masuda S: Clear cell carcinoid tumor of the gallbladder. A case without von HippelLindau disease. Arch Pathol Lab Med 127: 745-747, 2003.

9. Albores-Saavedra J, Nadji M, Henson DE, Ziegels-Weissman J and Mones JM: Intestinal metaplasia of the gallbladder: a morphologic and immunocytochemical study. Hum Pathol 17: 614-620, 1986.

10. Yamamoto M, Nakajo S, Miyoshi N, Nakai S and Tahara E: Endocrine cell carcinoma (carcinoid) of the gallbladder. Am J Surg Pathol 13: 292-302, 1989.

11. Sakamoto H, Mutoh H, Ido K, Satoh K, Hayakawa H and Sugano K: A close relationship between intestinal metaplasia and Cdx2 expression in human gallbladders with cholelithiasis. Hum Pathol 38: 66-71, 2007.

12. Maitra A, Tascilar M, Hruban RH, Offerhaus GJ and Albores-Saavedra J: Small cell carcinoma of the gallbladder: a clinicopathologic, immunohistochemical, and molecular pathology study of 12 cases. Am J Surg Pathol 25: 595-601, 2001.

13. Ishida M, Kushima R, Chano $\mathrm{T}$ and Okabe H: Immunohistochemical demonstration of the type III intermediate filament peripherin in human rectal mucosae and well-differentiated endocrine neoplasms. Oncol Rep 18: 633-637, 2007.

14. Ishida M, Kushima R, Brevet M, Chatelain D and Okabe H: Co-expression of neuronal intermediate filaments, peripherin and $\alpha$-internexin in human well-differentiated endocrine neoplasms (carcinoid tumors) of the appendix. Mol Med Report 1: 191-195, 2008. 